Service social

\title{
Le service social et les conditions de sa pratique : un rapport à examiner
}

\section{Marcelle Laforest et Belhassen Redjeb}

Volume 40, numéro 2, 1991

Formation et évolution de la pratique en travail social

URI : https://id.erudit.org/iderudit/706529ar

DOI : https://doi.org/10.7202/706529ar

Aller au sommaire du numéro

\section{Éditeur(s)}

École de service social de l'Université Laval

ISSN

1708-1734 (numérique)

Découvrir la revue

Citer cet article

Laforest, M. \& Redjeb, B. (1991). Le service social et les conditions de sa pratique : un rapport à examiner. Service social, 40(2), 89-104.

https://doi.org/10.7202/706529ar
Résumé de l'article

Les auteurs posent au départ que les conditions d'actualisation de la pratique du service social ont un impact sur le sens et la forme de cette pratique. Afin d'apprécier cette incidence de la condition sur la pratique, ils s'interrogent sur la dynamique qui régit le rapport entre les deux, plus particulièrement en contexte québécois. C'est ainsi que, dans un premier temps, ils dégagent les caractéristiques de chacune des conditions retenues, à savoir la technocratisation, la judiciarisation, la communautarisation et la privatisation de type lucratif, pour examiner, dans un deuxième temps, le rapport de chacune de ces conditions à la pratique du service social. Cet exercice de compréhension leur permet d'illustrer l'incidence des conditions des pratiques sur la pratique elle-même, selon qu'il s'agisse de conditions fortement étatisées ou de conditions émergentes et en marge de l'État. Ils concluent par une hypothèse sur l'état de la représentation du service social, hypothèse qu'ils induisent de la diversité observée de ses conditions de pratique. 
Marcelle Laforest, professeure à l'École de service social de l'Université Laval.

Belhassen Redjeb, professeur au Cégep de Lévis-Lauzon et chargé de cours à l'École de service social de I'Université Laval.

\title{
Le service social et les conditions de sa pratique : un rapport à examiner
}

\author{
Marcelle Laforest \\ Belhassen Redjeb
}

Que ce soit sous la pression de problématiques sociales nouvelles, d'innovations théoriques et méthodologiques ou de contextes de pratique variés, le service social est au carrefour des alternatives. De ce fait, il doit continuellement s'interroger, se réajuster et, s'il y a lieu, revoir les différentes configurations théoriques, normatives et méthodologiques qui le constituent en une pratique relativement autonome et sur laquelle repose l'identité professionnelle de ses acteurs. Comme tout champ qui se situe aux confins de la théorie et de l'action, le service social évolue à travers un processus de différenciation et d'intégration des données nouvelles aux données déjà organisées et intégrées. Et c'est l'une des tâches que se partagent les milieux de la formation et de la pratique que de procéder aux opérations de repérage, d'analyse et d'articulation qui sont à la base de ce processus toujours en mouvement de la construction du travail social.

$C^{\prime}$ est dans cette perspective que s'inscrit le présent article. Considérant que les conditions dans lesquelles s'actualise le service social ont une influence sur le sens et la forme que prendra l'acte de la pratique du service social dans un contexte et une conjoncture donnés, il nous apparaît pertinent et opportun de nous interroger sur la dynamique qui régit le rapport qu'entretient la pratique du service social avec les conditions dans lesquelles elle se déploie. L'exercice de compréhension que nous menons à cet effet n'a d'autres intentions que d'apporter notre modeste contribution à la réflexion suscitée par les enjeux contenus dans les différentes directions qui sollicitent 
actuellement l'évolution du service social et de participer ainsi à cette production du travail social dont nous faisions état plus haut.

L'analyse qui va suivre porte donc sur les conditions de la pratique du service social, plus particulièrement en contexte québécois, et sur les rapports qu'elles entretiennent avec ladite pratique. L'approche préconisée jette, dans un premier temps, un éclairage sur les termes de l'objet de la présente analyse pour ensuite s'attarder, dans un deuxième temps, à caractériser chacune des conditions de la pratique du service social. Ces conditions sont : la technocratisation, la judiciarisation, la privatisation de type lucratif et la communautarisation. Pour les caractériser, nous allons les envisager comme des « formes socia$l^{1} s^{1}$ » inspirées des différents courants de structuration du social qui actualisent les pratiques qu'elles engendrent, comme la gestion et la pratique judiciaire, et instruisent celles qu'elles abritent, en l'occurrence le service social. Après avoir ainsi démarqué les contours propres à chacune de ces conditions, nous examinerons le rapport de la condition étudiée à la pratique du service social, afin d'apprécier l'incidence de l'une sur l'autre. Nous conclurons, dans un troisième temps, par une hypothèse sur l'état de la représentation du service social, hypothèse induite de la diversité observée de ses conditions de pratique.

\section{La condition de la pratique : une double acception}

Considéré comme une pratique sociale médiatisée, donc redevable à un savoir-faire, le service social dépend de l'état d'avancement des connaissances cumulées sur son objet et les moyens d'intervenir sur cet objet. L'état des connaissances constitue, dès lors, une condition interne au service social, condition sur laquelle ses acteurs ont une prise. À cette première acception de la condition de la pratique, sur laquelle nous ne reviendrons qu'accessoirement dans le présent article, s'en ajoute une seconde qui renvoie, celle-là, à l'environnement à partir duquel l'intervenant social exerce son action. Le sens du terme condition se déplace ici vers celui de circonstance, mais garde l'idée de déterminant que toute condition présuppose. C'est cette acception du terme condition que nous retenons dans la présente analyse pour désigner les conditions externes de la pratique du service social. En effet, la technocratisation, la judiciarisation, la privatisation ainsi que la communautarisation sont des conditions externes au service social du fait que, d'une part, elles ne lui sont pas propres et que, d'autre part, elles sont irréductibles à des pratiques qu'elles organisent comme les professions des domaines social, psychologique et paramédical. En outre, ces conditions (même si c'est moins évident dans le cas de la 
communautarisation comme nous le verrons plus loin) sont issues de pratiques tout à fait autonomes, sans rapport obligé à celles qu'elles organisent.

En tant que cadres de travail instruits d'éléments contextuels, les conditions de la pratique correspondent à " des formes sociales institutionnalisées, différenciées, organisées ${ }^{2} »$, comme la technocratisation, la judiciarisation et la privatisation; ou en voie de l'être, comme la communautarisation. Une condition est institutionnalisée lorsqu'elle est établie, c'est-à-dire qu'elle a acquis " une forme matérielle ${ }^{3}$ ", donc une organisation, une "rationalité », une "fonctionnalité " et une légitimité à partir de " normes instituées ${ }^{4}$ ». Dès lors, pour observer et analyser chacune des conditions de la pratique du service social, trois dimensions nous semblent à la fois traduire les éléments de définition de la condition envisagée ici comme forme institutionnalisée ou en voie de le devenir et permettre de regrouper les attributs et caractéristiques propres à chacune des conditions soumises à l'étude. Ces dimensions sont donc, pour chaque condition entendue au sens de forme sociale organisée : un fonctionnement interne préconisé, un rapport à l'autre (notamment à la clientèle) envisagé et une légitimité mise de l'avant. Ce sont ces trois dimensions qui nous serviront de paramètres pour définir et analyser, dans ce qui suit, les contours de chacune des conditions dans lesquelles se déploie la pratique du service social.

\section{La technocratisation comme condition de pratique}

La technocratisation est cette condition de pratique qui correspond à l'instauration d'un type d'organisation caractérisé par le pouvoir déterminant que détiennent les techniciens-organisateurs dans l'orientation des choix des dirigeants ${ }^{5}$. Ces techniciens-organisateurs ou technocrates constituent ce que l'on désigne par le terme technocratie, laquelle représente le haut personnel qui occupe les postes de dirigeants de l'État et des entreprises privées ou publiques, postes qui leur confèrent un rôle politique prédominant qu'ils ne doivent ni à l'élection ni à un quelconque titre de propriété 6 . Selon cette conception, les technocrates seraient surtout perçus comme des techniciens qui ont accédé au pouvoir pour leurs capacités techniques, supplantant ainsi un personnel politique et gestionnaire traditionnel dépassé par la complexité des exigences scientifiques et techniques des sociétés modernes. Pour sa part, Morfaux ${ }^{7}$ soutient que les technocrates seraient moins des techniciens que des dirigeants et des coordonnateurs des différents techniciens, qui orientent les choix et les décisions de l'État et des organisations publiques ou privées à partir d'un 
ensemble de données techniques. Cette distinction donnerait lieu à une conception présentée par l'auteur comme étant plus positive que la précédente et correspondrait davantage au concept de management scientifique pour désigner la réalité technocratique et à celui de managers pour les experts remplissant les mêmes fonctions que celles dévolues aux technocrates dans la conception précédente. En réalité, la connotation plutôt péjorative souvent accolée au terme technocratie est due au fait que, dans les organisations où elle est installée, l'aspect technique et utilitaire a souvent priorité sur les considérations humaines et sociales. Quant à la connotation plus positive reliée au management scientifique, elle vient du fait que celui-ci préconise, dans la gestion des organisations, un type d'autorité fonctionnelle basée sur la recherche d'une coopération efficace entre tous les groupes qui composent l'organisation et constituent ce que Galbraith a désigné par le terme technostructure ${ }^{8}$. Ce bref rappel nous permet, à l'aide des paramètres annoncés ci-dessus, d'envisager la technocratisation en tant que condition de pratique.

La technocratisation repose sur un type de fonctionnement interne fortement structuré et où les acteurs se multiplient aussi bien sur le plan de l'encadrement du travail que sur le plan de la production des services. À cette division complexe du travail s'ajoute un code de procédures qui est utilisé au moment des transactions entre les unités de production. Cet outil est en quelque sorte un « lubrifiant " administratif pour ceux qui hésitent à faire le geste qu'il faut. Enfin, tous les actes importants traduisant le service sont consignés ou bien audit code de procédures ou bien aux autres manuels de circonstance. Ajoutons que la consigne à la base du fonctionnement technocratique est essentiellement productiviste en ce sens qu'elle privilégie le rapport du volume de production au temps de production, rapport instruit par la norme de la "prospérité maximale ${ }^{9}$ ".

Le rapport à la clientèle que postule le contexte technocratique est un rapport de besoin-réponse pour autant que les deux composantes sont interprétées ou interprétables de la même manière que ce qui est indiqué dans les programmes et les services. La qualité du rapport s'évalue davantage à son caractère bref et à son effet rapide et circonscrit qu'à sa profondeur et à sa portée immédiate et médiate. Enfin, le processus de prise en charge de la clientèle est parcellisé, atomisé plutôt que relevant d'un même acteur de l'organisation. D'où un rapport éphémère, utilitaire et souvent fruste au sens de non développé, sans raffinement thérapeutique et standardisé. Bref, ce sont des caractéristiques qui " tendent même à l'insensibilité vis-à-vis du consommateur ${ }^{10} »$.

La légitimité du contexte technocratique tient de deux référents idéologiques complémentaires et pouvant s'exprimer comme suit : 
1) reculer les frontières de l'aléatoire, prévenir et au besoin évacuer l'improvisation et I'anarchie dans toute hypothèse de production de services et 2) promouvoir l'ordre et le progrès (et le progrès de l'ordre) par la rationalité instrumentale et l'efficacité. C'est, en fait, la référence " à la maîtrise de la nature et à la productivité ${ }^{11} . .$. » qui fonde la légitimité de la technocratisation.

\section{Le rapport de la technocratisation à la pratique du service social}

L'intérêt principal et générique du technocrate est donc d'assurer son emprise sur la production des services de façon à satisfaire l'objectif de l'organisation dans laquelle se déploie son activité. Cet objectif est mis en forme dans des programmes conçus aux paliers supérieurs de l'organisation. II est de plus légitimé par un cadre légal, en l'occurrence au Québec, le chapitre 48 de la Loi sur la santé et les services sociaux, plus souvent désigné aujourd'hui par Loi S-5. Donc, le technocrate détient ainsi un mandat social qu'il réalise par des activités techno-gestionnaires, d'où le caractère impératif de la mission, caractère ayant un effet contraignant sur les rapports de l'organisation à son environnement.

C'est ainsi que le praticien du service social qui exerce dans ces organisations technocratiques subit ces impératifs qui ne sont pas sans marquer sa pratique, du moins dans l'image idéale (voire l'identité professionnelle) qu'il s'en fait. Le souci de se conformer à cette représentation d'où il tire un sens à sa pratique l'amène à exercer une pression du bas vers le haut. Ce qui engendre par le fait même un autre effet de contrôle qui rappelle à l'acteur-praticien " protestataire ou revendicateur " la mission d'ensemble de l'organisation. Cette mission est devenue (à cause de l'uniformisation et du souci d'efficacité) une sorte de "ce qu'il faut faire » qui se traduit, répétons-le, par des moyens qui prennent la forme de programmes, de manuels de procédures, d'une division du travail et autres règles destinées à assurer la réalisation de la mission en question.

Nous sommes, dès lors, en présence de deux groupes d'intérêts pas toujours conciliables : pour le technocrate-gestionnaire, il s'agit de préserver le contrôle sur l'organisation et, pour le praticien du service social, de préserver son identité professionnelle à travers sa pratique quotidienne au sein de ladite organisation. De plus, le premier acteur a un pouvoir légal et administratif, donc d'encadrement, et le second détient, en principe, une autorité sur l'acte d'intervention, autorité que lui confère un type de compétence professionnelle d'un intervenant employé et salarié de l'organisation et donc redevable à celle-ci. Il s'ensuit une dynamique dans laquelle, malgré le dialogue et 
la bonne volonté, les compromis ne se font souvent que dans un sens, celui des mandataires. La récurrence de cette dynamique et de son issue a sans doute un effet d'érosion sur le travail social envisagé comme profession avec ce que cela suppose comme perte d'autonomie relative de son acteur.

En somme, nous pouvons présumer que compte tenu des considérations analytiques que nous avons mises de l'avant, le travail social professionnel, dans ce contexte, s'actualise, au prix de l'intégrité de son identité, dans une réification à laquelle il participe par les concessions que ses acteurs font dans le quotidien de leur pratique, concessions qui vont dans le sens d'une déprofessionnalisation observée déjà au tout début des années soixante par $\mathrm{A}$. Etzioni au sein des organisations modernes américaines ${ }^{12}$.

\section{La judiciarisation comme condition de la pratique}

La judiciarisation, en tant que condition de la pratique du service social, constitue une des expressions de l'hégémonie du Droit en tant que trait caractéristique de la modernité ${ }^{13}$. Sa coexistence avec la technocratisation s'opère sur le terrain de la pensée utilitariste. Émanant de l'État, la judiciarisation est une mise en forme de « la contrainte inconditionnée $e^{14} »$. Pour sa part, elle contribue à instaurer un type de fonctionnement interne fortement codé et légalement formé. Dans ce contexte, « la règle de droit se répand de plus en plus à la manière des cellules cancéreuses, envahissant simultanément la sphère de la sociabilité spontanée et celle de la morale ${ }^{15} »$. Chartes, lois, règlements et infrastructure de circonstance sont donc caractéristiques de ce milieu qui a tendance à ne comprendre que son propre lexique et ceux qui en font l'usage. De plus, la pratique légale-judiciaire s'approprie le social et le réécrit, ce qui donne lieu à " une inflation législative et judiciaire " pour reprendre l'expression de Jacques Dufresne. L'interprétation de la situation en vue de la production du service à offrir ou à imposer se fait à partir du prisme légal-judiciaire.

Le rapport à la clientèle envisagé par la judiciarisation se noue à la rencontre d'un social qui est objet de légalité et d'une procédure réglementée. Spécialiste et profane sont donc soumis à l'interprétation judiciaire, quand ils n'en appellent pas eux-mêmes. En outre, la tendance marquée de l'État à traiter les citoyens en fonction d'une stricte légalité ${ }^{16} n^{\prime}$ induit $d^{\prime}$ aucune manière l'absence d'une conception légalejudiciaire de la socialité. Au contraire, le légal-judiciaire construit une socialité en se construisant, socialité par laquelle il instruit le social plus souvent qu'il ne s'en inspire. La judiciarisation est, de ce point de vue, porteuse d'un projet de socialité qu'elle érige en contrainte " en tant 
que fixant des limites sur l'éventail d'options dont dispose un acteur ou un ensemble d'acteurs, dans un contexte donné ou dans un type de contexte $^{17}$ ". De ce fait, la judiciarisation est une condition d'autorité à laquelle l'intervenant social et le citoyen visé par l'intervention socialejudiciaire sont tenus de se soumettre.

La légitimité de la judiciarisation repose, en dernière analyse, sur les " méga-lois " que sont les chartes de droits, source intarissable de motivations et de motifs pour les législateurs et la pratique judiciaire. Et comme les chartes s'appuient sur la défense et la protection des droits de la personne, le discours qu'elles tiennent se trouve à être à la fois la source et la finalité de la judiciarisation. Cette légitimité est d'autant moins contestée que la "normativité sociale " est nébuleuse du fait de la modernité.

\section{Le rapport de la judiciarisation à la pratique du service social}

L'acteur au service du cadre judiciaire, donc agissant dans et sous cette condition, engage avec l'acteur ciblé, parce qu'interpellé par la loi, un rapport plutôt légal, parfois à visage social. Volontaire ou non, ce rapport est régi par une série d'articles de lois et de règlements qui constituent en fait des limites et des balises dans l'engagement de l'un ou de l'autre de ces acteurs, et ce, en procédant à la " définition des règles qui doivent régir la relation entre le professionnel et son bénéficiaire (et à la) définition du processus par lequel doit passer l'acte professionnel ${ }^{18}$ ».

Ici, l'impératif légal, qui s'ajoute à l'impératif gestionnaire, transcende la pratique du service social et, de ce fait, l'oriente à partir de ce que le législateur a conçu comme projet d'action sociale. Ainsi, le diagnostic, prérogative professionnelle par excellence, est ici érigé en articles de loi auxquels le praticien fait correspondre ses interventions. $C^{\prime}$ est ainsi que sous la condition de la judiciarisation, le praticien est appelé à utiliser de plus en plus un savoir procédural, appris «sur le tas " aux dépens du savoir analytique et méthodologique acquis au cours de sa formation initiale en service social.

La pratique du service social, sous la condition de la judiciarisation, subit ainsi des transformations qui se superposent à celles introduites par la technocratisation. Cette double détermination, redevable à la fois à la contrainte légale-judiciaire et à l'impératif gestionnaire, n'est pas sans introduire non seulement une tout autre structuration de l'acte de ladite pratique, mais aussi et surtout un déplacement de ses préoccupations relevant de la régulation sociale vers d'autres qui sont plutôt de nature tutélaire. Déjà, en 1980, F. Lesemann et G. Renaud concluaient en ce sens une étude portant sur le rapport entre la 
Loi sur la protection de la jeunesse et les pratiques professionnelles en service social ${ }^{19}$.

Les deux conditions de la pratique en service social que nous venons de décrire à gros traits sont nettement prédominantes dans la sphère étatique et elles coexistent sour forme de tandem légal-rationnel d'organisations étatiques de services. Ce tandem s'est édifié autour de "l'activité rationnelle par rapport à une $\operatorname{fin}^{20}$ " que les acteurs (praticiens et bénéficiaires) n'ont pas à définir, mais auxquels ils sont différemment assujettis.

Les deux autres conditions que nous traitons ci-après, à savoir la communautarisation et la privatisation de type lucratif, se situent en marge de la sphère étatique avec laquelle elles entretiennent un rapport plus ou moins proche, rapport souvent justifié par leurs propres stratégies de survie.

\section{La communautarisation comme condition de la pratique}

La communautarisation est une forme sociale «bipolaire " où l'on note la présence, d'un côté, de pratiques provenant " de la libre association en marge ou à la marge du contrôle direct des entreprises de l'État et (...) de l'Église ${ }^{21}$ " et, de l'autre côté, de pratiques mises en œuvre ou investies par ces mêmes acteurs dans un espace communautaire. Alors que les premières pratiques sont redevables à des solidarités choisies et volontaires ${ }^{22}$, les secondes sont suscitées à partir de préoccupations strictement utilitaristes ${ }^{23}$. Bien entendu, cette bipolarité a une incidence sur la nature de la pratique du travail social. Toutefois, I'angle d'analyse privilégié ici permet de considérer la communautarisation en tant qu'une condition de pratique englobante et qui renvoie, selon le cas, aux conceptions du communautaire mentionnées plus tôt.

Le type de fonctionnement interne que préconise la communautarisation, en tant que contexte de.pratique, est caractérisé par une organisation allant du simple au précaire étant donné les rapports inconstants de tous genres qu'elle entretient avec l'extérieur (État ou sources d'aide privées). Souvent volontaires, parfois sous-rémunérés, les acteurs de ces organisations se divisent le travail de coordination et le travail de terrain sans pour autant banaliser de façon étanche cette division du travail. De façon générale, les règles de fonctionnement pour produire le service ne font pas l'objet de manuels, de modes d'emploi et elles se limitent à des consignes tantôt écrites, tantôt orales. Enfin, le service à produire est peu spécialisé, peu bureaucra- 
tisé et il incite au contact régulier avec le milieu; d'où ce caractère communautaire ${ }^{24}$.

Le rapport à la clientèle prend des formes d'expression qui tiennent du volontariat ancien ou nouveau, les deux qualificatifs étant, bien entendu, pris dans leur sens relatif, ou du militantisme. Ainsi on peut dégager dans cette condition de pratique un premier rapport, repérable surtout dans les organisations bénévoles de type traditionnel, où l'acteur producteur du service est une personne dévouée et où l'acteur qui reçoit le service est une personne nécessiteuse. Un second rapport, que l'on retrouve dans des organisations du genre maisons $d^{\prime}$ hébergement pour femmes violentées par exemple, est un rapport où l'acteur producteur du service est le plus souvent professionnel (dévoué dans les circonstances puisque, s'il n'est pas bénévole, il est au moins sous-rétribué) et l'acteur recevant le service est considéré comme étant un bénéficiaire. Une autre variante est celle où l'acteur produisant le service est ou était l'acteur recevant ou ayant reçu le service; dans ce cas, l'assistant est assisté et l'assisté est assistant. II s'agit d'un rapport d'aide mutuelle que l'on observe dans les groupes $d^{\prime}$ entraide. Le troisième rapport est explicitement marqué par une solidarité entre "les intervenants du monde communautaire ${ }^{25}$ " et les autres membres d'une communauté. Cette solidarité est alignée sur des enjeux sociaux qui dépassent la simple préoccupation de service sans pour autant l'exclure.

Dans l'ensemble, le rapport à la clientèle dans le cadre de la communautarisation prend la forme ou le type que le milieu veut bien lui donner. Il est toutefois à noter que, dans plusieurs cas, les pressions exercées par les organismes pourvoyeurs ou subventionnaires (étatiques ou non) pour se conformer aux règles et standards de la gestion limitent cette autonomie.

La légitimité de ce contexte de pratique se nourrit pour ainsi dire de la critique néo-libérale pour le communautaire de services et de la critique sociale-démocrate pour ce qui est du communautaire mili$\operatorname{tant}^{26}$. La première $\mathrm{s}^{\prime}$ applique à remettre en cause l'omniprésence de la technique et du technicien dans l'ordonnancement du quotidien ${ }^{27}$; l'essentiel de son argumentation peut se résumer par cette phrase célèbre de Georges Bernanos : "Un monde gagné pour la technique est perdu pour la liberté ". Le retour à l'humain apparaît donc comme le salut, comme une sorte d'issue à la marche infernale vers ce qui s'apparente à la production de l'humain "sous vide ". Cette même critique s'élève contre l'omnipotence de l'État dans la sphère sociale, omnipotence manifestée par une prise en charge publique sans précédent dans le monde occidental et dont le coût financier est d'autant plus élevé que les pertes de solidarité naturelle que cette prise en charge entraîne sont considérables. Dès lors, le recours à la prise en 
charge dite par le milieu apparait comme inexorable et, curieusement, la responsabilisation du milieu devient, à cet égard, le projet numéro un de l'État. En somme, le retour à l'humain et aux bienfaits du milieu naturel (humanisation et solidarité naturelle) constitue le bien-fondé de la communautarisation telle qu'envisagée par la critique néo-libérale.

La critique sociale-démocrate et militante prend le contre-pied de la critique néo-libérale au sujet de la communautarisation en rejetant la préoccupation " utilitariste " et en mettant de l'avant la nécessité d'une "identité communautaire " pour disposer de celles que les institutions en place imposent ${ }^{28}$.

\section{Le rapport de la communautarisation à la pratique du service social}

À la différence des conditions de pratique précédentes, la communautarisation est celle à l'édification de laquelle l'intervenant participe, en ce sens que sa pratique informe, au sens plein du terme, le cadre dans lequel elle se déploie et, dans certains cas, le détermine. En revanche, ce même cadre peut, dans des contextes observés, instruire la pratique et la rendre reproductible ${ }^{29}$. En somme, ici, la condition de la pratique et la pratique elle-même s'engendrent l'une l'autre tant il est vrai que les deux n'ont de sens qu'ensemble.

Dans la communautarisation, l'intérêt des acteurs de la " prise en charge ", et notamment de l'intervenant social, est d'abord et avant tout de rechercher une certaine originalité que lui suggère sa compétence particulière, lorsque celle-ci est soumise à l'épreuve du problème à résoudre. C'est aussi de faire ce qui ne peut être fait dans les organisations modernes d'État où tout est pratiquement défini à l'avance. C'est en somme vivre autrement son identité professionnelle ou militante avec une place plus grande à l'initiative. Le contexte communautarisé apparaît dans cette perspective comme un élément favorable à l'actualisation de l'intervention en service social, notamment en organisation communautaire.

Mais, il est vrai que cette quête de l'autrement et de l'autonomie matérielle a l'inconvénient de soumettre la pratique aux aléas de l'improvisé et de l'incertitude matérielle. À telle enseigne que des organismes de prise en charge communautaire deviennent vulnérables et, de ce fait, susceptibles d'être eux-mêmes pris en charge d'une part par $l^{\prime}$ expert ${ }^{30}$ et le gestionnaire pour voir à ce qui leur paraît problématique et, d'autre part, par l'État qui peut, à l'aide de subventions, diminuer les incertitudes matérielles en échange d'un droit de regard sur les finalités de l'action et, parfois, sur la performance des acteurs de la pratique $^{31}$. 


\section{La privatisation de type lucratif comme condition de la pratique}

La privatisation de type lucratif, ou pratique en bureau privé, représente un cadre de travail qui va croissant depuis quelques années pour la pratique du service social. D'où l'importance de nous arrêter ici à examiner cette quatrième condition de la pratique du service social. Son fonctionnement interne s'articulant sur celui du marché des services (impliquant l'offre et la demande) préconise la concurrence, l'émulation et des honoraires compétitifs. L'organisation pour la production du service est plutôt simple et peu élaborée. De plus, la production du service revient souvent à l'acteur qui amorce la prise en charge en intervention sociale.

Le rapport à la clientèle est un rapport de producteur de service à consommateur de service. En principe, le praticien contracte une " obligation de moyen » envers la clientèle qui verse des honoraires en retour. Cette caractéristique est absente dans les autres conditions de pratique. De plus, le client, payant directement le service, est porté à mieux en scruter la qualité et à mettre davantage sa volonté à contribution dans la réussite de ce service.

La privatisation de type lucratif assoit sa légitimité sur deux types de considérations, à certains égards complémentaires. Les premières sont normatives, comme la liberté d'initiative et d'entreprise, ainsi qu'une éthique relevant des professions libérales connues. Quant aux secondes, elles tiennent plutôt du rejet de l'étatisme bureaucratique.

\section{Le rapport de la privatisation à la pratique du service social}

Sous la condition de la privatisation de type lucratif, le praticien fait face aux contraintes rattachées au marché des services, largement occupé par des pratiques dont l'objet avoisine celui du service social quand ce n'est pas le même. Or, il est connu que le caractère thérapeutique de ces services s'adressant à des individus, couples et familles domine nettement leur prestation. L'objet de la pratique est plutôt défini par le marché, mais en même temps on sait que dans sa formulation idéale le service social accorde une place de choix à une pratique dont le concept organisateur est «la personne en situation ${ }^{32}$ ". Tout se passe donc comme si la privatisation de type lucratif permettait l'actualisation d'une représentation professionnelle de la pratique du service social, représentation acquise par les praticiens, il va sans dire, au moment de la formation initiale. L'intérêt récent et grandissant pour la pratique privée $e^{33}$ ne fait que corroborer cette observation. 
Concrètement, l'intérêt du praticien travaillant dans ce cadre est de différents ordres. Ainsi, dans ce contexte, il contrôle sa pratique et il lui est possible de se doter d'une réputation professionnelle (moins facile à obtenir dans les autres conditions) conforme à l'effort qu'il déploie et donc, de se réaliser personnellement et socialement en obtenant le statut qu'il estime mériter. Ce cadre de pratique lui permet aussi de prendre une part du marché, soit dans la clientèle des mieux nantis, soit dans la " clientèle subventionnée ». Ici, le travailleur social de pratique privée s'entend avec les services sociaux de l'État pour obtenir une clientèle que lesdits services ne peuvent plus desservir (ex. : clientèle qui demande des services spécialisés, comme des services de counseling conjugal). On pourrait voir ainsi une certaine possibilité pour le travailleur social de substituer au rôle de commis de l'État celui de partenaire de l'État. Par ailleurs, I'accroissement de l'effectif des travailleurs sociaux de pratique privée des dernières années pourrait se heurter à une concurrence des professionnels du champ des autres thérapeutes. Cette lutte pourrait conduire le travailleur social à réexaminer son identité propre pour se rapprocher de ces pratiques, s'éloignant ainsi de la représentation idéale du service social ${ }^{34}$.

\section{Conclusion}

Nous posions, au départ, que les conditions d'actualisation de la pratique du service social ont une influence sur le sens et la forme que prendra celle-ci. Afin d'apprécier cette incidence de la condition sur la pratique, nous nous sommes interrogés sur la dynamique qui régit le rapport entre les deux. C'est ainsi que, pour chacune des conditions retenues, nous avons, en nous inspirant du cas du Québec, dégagé dans un premier temps les caractéristiques à l'aide de paramètres énoncés au préalable pour, dans un deuxième temps, examiner le rapport conséquent de chaque condition à la pratique du service social. Cet exercice de compréhension a permis d'illustrer l'incidence des conditions des pratiques sur la pratique elle-même, selon qu'il $s^{\prime}$ agit de conditions fortement étatisées ou de conditions émergentes et en marge de l'État. Pour conclure, nous commençons, ci-après, par résumer, sur le plan des répercussions, les résultats de nos analyses quant à l'impact des conditions de pratique sur la pratique même. Par la suite, nous formulons des hypothèses d'observation à propos de la diversification de ces conditions de la pratique et de leur incidence sur cette dernière.

Ainsi, les deux premières conditions de la pratique en service social que nous avons étudiées, à savoir la technocratisation et la 
judiciarisation, sont nettement prédominantes dans la sphère étatique et elles coexistent sous forme de tandem légal-rationnel d'organisations étatiques de services. Ce tandem s'est édifié autour de «I'activité rationnelle par rapport à une fin ${ }^{35}$ " que les acteurs (praticiens et bénéficiaires) n'ont pas à définir, mais à laquelle ils sont différemment assujettis. C'est ainsi que nous avons vu que la technocratisation des services sociaux avait tendance à mettre de l'avant des conditions qui mènent à la déqualification et à la déprofessionnalisation des diverses pratiques qu'elle englobe, dont en l'occurrence ici le service social. Nous avons aussi constaté que la judiciarisation contribue à instaurer des éléments contextuels structurants qui sont de l'ordre de l'impératif légal-judiciaire et qui, superposés à ceux de la technocratisation, en arrivent à exercer un contrôle quasi absolu sur le déroulement de la pratique du service social. II s'agit ici d'un contrôle qui, non seulement, déqualifie ladite pratique, mais la soumet en quelque sorte à une mise en tutelle compte tenu des limites exercées sur la marge d'autonomie de l'intervenant, laquelle marge d'autonomie est une caractéristique admise et importante dans la représentation professionnelle du service social.

Les deux autres conditions étudiées, la communautarisation et la privatisation de type lucratif, se situent, quant à elles, en marge de la sphère étatique avec laquelle elles entretiennent un rapport plus ou moins proche, rapport le plus souvent justifié par leurs propres stratégies de survie. Dans un contexte communautarisé, l'intervenant participe à l'édification des éléments contextuels qui deviennent de ce fait imbriqués à sa pratique même; dans cette perspective, la condition apparaît comme un élément favorable à l'actualisation de la pratique de service social prise dans ses dimensions idéales. Cependant, comme nous l'avons vu plus haut, la marge d'autonomie, dans certains cas, risque d'être le prix à payer pour échapper aux aléas de l'improvisé et de l'incertitude matérielle qui sont trop souvent le lot des organismes communautaires. Par ailleurs, le contexte de privatisation se révèle favorable à l'actualisation de la représentation idéale de la pratique du service social, notamment en ce qui concerne la marge de manœuvre. Les lois du marché dans lesquelles s'inscrit ce type de pratique risquent cependant d'amener le praticien à revoir son identité de travailleur social pour se rapprocher des pratiques concurrentes qui occupent le champ de la thérapie.

La diversité des conditions de la pratique du service social dont nous avons fait état $n$ 'induit pas que celles-ci s'affirment avec la même intensité dans la structuration de ladite pratique. En réalité, la technocratisation et la judiciarisation sont d'autant plus affirmées que l'État en est le commanditaire; ce qui ne semble pas le cas quand on observe les autres conditions de la pratique du service social et ce, pour des 
raisons différentes. En effet, la communautarisation, bien que montante, demeure relativement marginale en raison de ses sources de soutien disparates. Quant à la privatisation, malgré une augmentation sensible de ses adeptes, elle reste l'apanage des clients qui peuvent se la payer ou qui sont assistés par l'État.

La diversité des conditions de la pratique du service social induit, par ailleurs, une diversification de la pratique proprement dite, compte tenu de l'incidence établie plus haut des unes sur l'autre. C'est sur la base de cette observation qu'il est permis de fonder l'hypothèse selon laquelle il y a une double représentation de la pratique du service social. La première s'édifie autour d'une pratique plutôt assujettie au contrôle étatique de type techno-judiciaire. Cette pratique participe à une mission de protection sociale de laquelle elle tire sens et substance. Dans ce cas, le travail social est une activité instrumentale " rationnelle par rapport à une fin $^{36}$ " dont il est redevable et à la définition de laquelle l'intervenant social ne participe pas. Dans cette perspective, l'intervenant est, malgré les apparences que la rationalité technique rend possibles, un mandataire agisssant pour un mandant qui est l'État et dont l'activité est destinée à un sujet interpellé. Cette représentation fait du service social une sociotechnique déployée dans le cadre d'une régulation (techno-judiciaire) fortement codifiée à l'avance.

La seconde représentation de la pratique du service social peut être inférée du travail social déployé dans les conditions de la communautarisation ou de la privatisation. Dans ces deux cas, le travail social est une activité communicationnelle, donc une interaction, par laquelle deux sujets agissants recherchent un consensus à propos d'une action. L'intersubjectivité et le dialogue qui met cette dernière en œuvre procèdent d'une " rationalité pratique ${ }^{37}$ » orientée par des normes reconnues par les deux sujets et préoccupée par la réussite de la démarche de formation d'une volonté d'action. Cette représentation se rapproche aisément de la conception du service social telle qu'elle se dégage des écrits qui traduisent les fondements du service social.

La double représentation du service social que l'analyse de ses conditions de pratique a permis d'inférer rend péremptoire un questionnement, à venir, quant au statut du savoir et à la normativité du service social. Ce questionnement ne peut désormais ignorer les conditions dans lesquelles la pratique s'exerce et les acteurs de la discipline ne peuvent plus en faire l'économie. 


\section{Notes}

${ }^{1}$ George Simmel, Sociologie et épistémologie, Paris, Presses universitaires de France, 1981, p. 171-206. Georges Lapassade et René Loureau, Clefs pour la sociologie, Paris, Seghers, 1974, p. 161 et 181.

${ }^{2}$ G. Lapassade et R. Loureau, ibid., p. 161 et 181.

${ }^{3}$ Ibid., p. 181.

${ }^{4}$ Idem.

${ }^{5}$ Jacques Billy, Les technocrates, Paris, Presses universitaires de France, Que sais-je ?, 1975, p. 5-8.

${ }^{6}$ La Sociologie, tome 3, Paris, Marabout Université, 1972, p. 663.

${ }^{7}$ Louis-Marie Morfaux, Vocabulaire de la philosophie et des sciences humaines, Paris, Librairie Armand-Colin, 1980, p. 359.

${ }^{8}$ Idem, p. 359 et 203.

${ }^{9}$ F.W. Taylor, Principes d'organisation scientifique dessinés dans Francine Séguin-Bernard et Jean-François Chaulat, L'analyse des organisations, une méthodologie sociologique, tome 1, Les théories de l'organisation, SaintJean-sur-Richelieu, Éditions Préfontaine inc., 1983, p. 79-118.

${ }^{10}$ Amitai Etzioni, Les organisation modernes, Gembloux, Duculot, 1971, p. 178.

${ }^{11}$ Herbert Marcuse, cité dans Jürgen Habermas, La technique et la science comme idéologie, Paris, Denoël/Gonthier, 1978, p. 7.

${ }^{12}$ Amitai Etzioni, op. cit., p. 161.

${ }^{13}$ Guy Rocher, "La bioéthique comme processus de régulation sociale : le point de vue de la sociologie ", dans Bioéthique : Méthodes et fondements, publié sous la direction de Marie-Hélène Parizeau, Faculté de philosophie de I'Université Laval, distribué par I'ACFAS, 1989, p. 52.

${ }^{14}$ Jean-Guy Belley, " L'État et la régulation juridique des sociétés globales : pour une problématique du pluralisme juridique ", dans Sociologie et sociétés, PUM, vol. XVIII, nº 1, avril 1986, p. 15.

${ }^{15}$ Jacques Dufresne, Le procès du droit, Québec, Institut québécois de recherche sur la culture, 1987, p. 54.

${ }^{16}$ François Châtelet, et al., "L'État-gérant ", dans Histoire des idées politiques, Paris, Presses universitaires de France, Coll. Thémis, 1982, p. 132.

${ }^{17}$ Anthony Giddens, La constitution de la société, Paris, Presses universitaires de France, 1987, p. 235.

${ }^{18}$ Jean-Pierre Duplantie, "Affirmer notre richesse ", Intervention, revue de la CPTSQ, n 72 , septembre 1985, p. 7.

${ }^{19}$ Frédéric Lesemann et Gilbert Renaud, "Loi 24 et transformation des pratiques professionnelles en service social », Intervention, n 58 , été 1980, p. 57.

${ }^{20}$ Jürgen Habermas, La technique et la science comme idéologie, Paris, Denoël/ Gonthier, 1978, p. 5.

${ }^{21}$ Gérald Doré, Partenariat : I'enjeu de la " coopération conflictuelle " pour les groupes communautaires, Laboratoire de recherche, École de service social, Université Laval, janvier 1991, p. 3.

${ }^{22}$ Ibid.

${ }^{23}$ Ibid. 
${ }^{24}$ Marcelle Laforest et Belhassen Redjeb, L'intervention sociale non salariée : étude exploratoire de dix pratiques d'assistance bénévole auprès des personnes âgées dans la région de Québec, Laboratoire de recherche de l'Eccole de service social, Université Laval, Sainte-Foy (Québec), 1989, p. 91-103.

${ }^{25}$ Gérald Doré, op. cit., p. 3.

${ }^{26}$ Idem, p. 2 et 3.

${ }^{27}$ Conseil international de l'action sociale, région Europe, La crise économique de l'État-Providence : conséquences et nouvelles approches dans les pays européens, contribution à la XXIII ${ }^{\text {e }}$ conférence internationale du Conseil international de l'action sociale, Montréal, éd. : Association allemande pour I'assistance publique et privée, août 1984.

${ }^{28}$ Gérald Doré, op. cit., p. 1-9.

${ }^{29}$ Marcelle Laforest et Belhassen Redjeb, op. cit., p. 115-121.

${ }^{30}$ Belhassen Redjeb, "L'expert ou la sagesse mutilée ", dans Les Cahiers, Revue d'essai et de littérature, Québec, Collège de Limoilou, vol. 1, $\mathrm{n}^{\circ} 1$, printemps 1991, p. 183-200.

${ }^{31}$ Marcelle Laforest et Belhassen Redjeb. op. cit., p. 115-121.

${ }^{32}$ À titre d'exemples, Helen H. Perlman, L'aide psychosociale interpersonnelle, Paris, Le Centurion, 1972, p. 16-36 (traduction de Social Casework, a ProblemSolving Process, University of Chicago Press, 1957), Florence Hollis, Casework, A Psychosocial Therapy, New York, Random House, 1965, p. 9-32, B. Compton et B. Gallaway, Social Work Processes, The Dorsey Press, 1984, p. 1-32, Lawrence Shulman, The Skills of Helping Individuals and Groups, F.E. Peacock Publishers, Itasca, III., 1984.

${ }^{33}$ Marie Sénécal-Émond, "Le travailleur social et la pratique privée ", Revue canadienne de service social 84, 1984, p. 214-219.

${ }^{34}$ Johanne Fillion, "Le service social est-il plus qu'un lieu ? ou référeriez-vous à un travailleur social en bureau privé ", document miméo., produit dans le cadre du Séminaire sur les conditions de la pratique, École de service social, Université Laval, Québec, 1988, p. 31-35.

${ }^{35}$ Habermas, Jürgen, op. cit., p. 5.

${ }^{36}$ Idem, p. 21.

${ }^{37}$ Idem, p. 22 et 23. 